\title{
Quantifying the Traction Force of a Single Cell by Aligned Silicon Nanowire Array
}

\author{
Zhou Li,, ,‡ Jinhui Song, ${ }^{\ddagger}$ Giulia Mantini,,, Ming-Yen Lu,, ,ll Hao Fang, ${ }^{\ddagger}$ \\ Christian Falconi, ${ }^{\S}$ Lih-Juann Chen," and Zhong Lin Wang ${ }^{\star, \neq}$ \\ Department of Advanced Materials and Nanotechnology, College of Engineering, \\ Peking University, 100084 Beijing, China, School Materials Science and Engineering, \\ Georgia Institute of Technology, Atlanta, Georgia 30332-0245, Department of \\ Electronic Engineering, University of Tor Vergata, Via del Politecnico 1, 00133 Roma, \\ Italy, and Department of Materials Science and Engineering, National Tsing Hua \\ University, Hsinchu, Taiwan 30043, R.O.C.
}

Received June 3, 2009

\begin{abstract}
The physical behaviors of stationary cells, such as the morphology, motility, adhesion, anchorage, invasion and metastasis, are likely to be important for governing their biological characteristics. A change in the physical properties of mammalian cells could be an indication of disease. In this paper, we present a silicon-nanowire-array based technique for quantifying the mechanical behavior of single cells representing three distinct groups: normal mammalian cells, benign cells (L929), and malignant cells (HeLa). By culturing the cells on top of NW arrays, the maximum traction forces of two different tumor cells (HeLa, L929) have been measured by quantitatively analyzing the bending of the nanowires. The cancer cell exhibits a larger traction force than the normal cell by $\sim 20 \%$ for a HeLa cell and $\sim 50 \%$ for a L929 cell. The traction forces have been measured for the L929 cells and mechanocytes as a function of culture time. The relationship between cells extending area and their traction force has been investigated. Our study is likely important for studying the mechanical properties of single cells and their migration characteristics, possibly providing a new cellular level diagnostic technique.
\end{abstract}

A change in the physical properties of mammalian cells is important for understanding the biological behavior of cells and disease diagnosis. ${ }^{1-3}$ Some kinds of malignant cells are difficult to diagnose from normal cells just by morphological analysis, which is a routine cancer cell detection method. The physical properties of stationary cells, especially the cell traction force (CTF), are of special interest because they are crucial to many biological processes such as morphology, extracellular matrix (ECM) generation, mechanical signal transmission, and cell migration. The measurements are potentially useful for understanding the processes of wound healing, inflammation, embryogenesis, angiogenesis, histogenesis, invasion, and cancer metastasis. ${ }^{4-7}$

The basic process for cells to generate traction force is the actomyosin tension and actin polymerization that contract the cell body and stress fibers, which transmit the constriction force to ECM via focal adhesion proteins. ${ }^{3,6}$ In an assembly of focal adhesion proteins, integrins are the primary mediators

\footnotetext{
* To whom correspondence should be addressed.E-mail: zlwang@ gatech.edu.

$\dagger$ Peking University.

* Georgia Institute of Technology.

$\S$ University of Tor Vergata.

"National Tsing Hua University.
}

that connect the actin cytoskeleton and ECM to provide a physical linkage (Figure 1). ${ }^{8-12}$ Hence, they are the adsorptive proteins with substrate and play a central role in cellular mechanical force transduction. As we know from literatures, ${ }^{8,13,14}$ stationary cells in suspended medium anchorage to flat substrate will first extend to a wider area under gravitation, come in contact with the supporter underneath, and then sense and build physiochemical linkage for focal adhesion. When cells finish organizing the ECM and complete the mechanical signal transmission, they begin to contract asymmetrically and start to migrate. Quantitative analysis of the mechanical behavior of cell traction force is an area of intensive study with copious challenges. ${ }^{1,3,7}$

Various reports about the physical characteristics of mammalian cells have been published in recent years. Cell stiffness has been suggested as a method to diagnose cancer cells. ${ }^{2}$ The mechanism for regulating cell adhesion and the molecular signal pathway under conditions of variable mechanical stress has been studied by atomic force microscopy (AFM) ${ }^{15}$ Several different methods have been developed for measuring CTFs. Polymerized bovine collagen was mixed with cells to form a gel disk that monitors the cell 


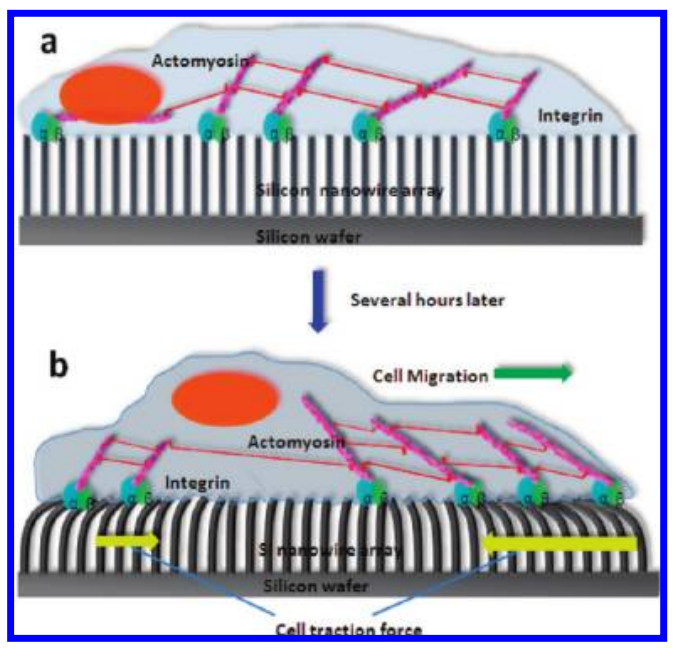

Figure 1. Schematic diagrams showing cell adhesion to aligned Si-NW array via focal adhesion and the traction force generated through the connective motivation of actin cytoskeleton, focal adhesion and extracellular matrix. (a) Cell attached with Si NW arrays for a short period of time. Multimolecular assemblies link the extracellular matrix to the cytoskeleton of the cell via membranebound receptors. Specific adhesion molecules such as integrins $(\alpha$, $\beta$ ) are therefore also the sites at which forces are transmitted to the substrate. (b) Cell begins to migrate along a direction and bend the $\mathrm{Si}-\mathrm{NWs}$ underneath as an action of traction forces. The traction forces distribute asymmetrically to form a resultant force pointing at the migration direction and at the same time to overcome the tendency of the cell extending and thinning in order to maintain the cell's integrality and mechanical stability. The higher the density and resolution of the NWs beneath the cell, the more information we can extract from the cell about its traction force as well as its interaction with substrate and ECM.

force, ${ }^{3,16}$ but it is unable to measure the CTF of individual cells. Thin silicone film provides another method for CTF measurements, ${ }^{17}$ but the data analysis is rather complex owing to the involvement of nonlinear mechanics for quantifying the wrinkles of the thin film. A micropost force sensor array has been developed and widely used for measuring CTF. ${ }^{16}$ From thousands of microposts, local traction force generated by cells can be measured along all directions for a single cell. ${ }^{5,14}$ The diameter of the microposts is $>0.75 \mu \mathrm{m}$ and the length is adjustable. ${ }^{20,21}$ The long micropost produces very flexible, extendable and soft beams that are easily bent, so that the measured force may not be the maximum force applied by the cell, while a short micropost can suffer from elastic elongation (or the pulling up effect) of the substrate and itself. As a result, the accuracy of the measured force may be limited by two factors, one being the density of the microposts and the other the resolution of the optical measurement technique adopted. ${ }^{22-24}$

Silicon nanowires ( $\mathrm{Si}-\mathrm{NWs}$ ) have been demonstrated as unique probes for quantifying biological processes at a high spatial resolution. An integration of arrays of field effect transistors made using Si-NWs and the neuron system by Lieber presents a breakthrough approach for studying electrical signal transmissions in neuron and biological species. ${ }^{25-30}$ In this paper, by culturing cancer cells and normal cells on Si-NW arrays, respectively, the deflections of the NWs were used to derive the lateral cell CTFs in reference to the calibrated force-displacement curve received by atomic force microscopy and finite element simulation. Our results indicate that the cancer cells (HeLa, L929) have significantly larger CTFs than a normal cell, and both of them in the microNewton range. This study presents a nanowire-based methodology for quantifying the mechanical behavior of single cells, which could be useful for understanding the migration behavior of cells.

Sample Preparation. To investigate the maximum CTF that can be generated from a single cell, uniform Si NW arrays with controlled diameters have been fabricated by an aqueous electroless chemical etching of silicon wafers (Supporting Information) using a published procedure. ${ }^{31}$ The diameter, length, and uniformity of the Si NWs were well controlled by etching conditions.

Three cell lines were used as model systems in this study, mechanocytes, L929 cells, and HeLa cells. Mechanocytes were harvested from neonatal rat liver (rat breed: Hsd: NSA, CF-1) by trypsinase digested. As it was fresh and never cultured in vitro, it played a role as the normal cells for this study. L929 cells and HeLa cells were purchased from American type Culture Collection (ATCC). They were employed as benign cells and malignant cells. Mechanocytes were cultured in RPMI-1640 medium (ATCC) supplemented with 10\% fetal bovine serum (FBS, ATCC) and 1\% penicillin/streptomycin (ATCC). L929 and HeLa cell lines were cultured in Dulbecco's modified Eagle's medium (DMEM, ATCC) supplemented with 10\% fetal bovine serum (FBS, ATCC) and 1\% penicillin/streptomycin (ATCC). Three different cell lines were incubated at $37{ }^{\circ} \mathrm{C}$ in $5 \% \mathrm{CO}_{2}$ and at a constant humidity.

The aligned Si-NW arrays were sterilized by immergence in $75 \%$ medical standard ethanol for $2 \mathrm{~h}$ and then exposure to a high-dose UV light for $2 \mathrm{~h}$. After sterilization, the aligned Si-NW arrays were rinsed by deionized water (DI water) 3 times and dried using extra pure nitrogen gas.

Suspended cells were prepared from anchorage cells cultured in vials for 3 days. The anchorage cells were treated with $0.05 \%$ trypsinase $2 \mathrm{~mL}$ for $90 \mathrm{~s}$ in $37^{\circ} \mathrm{C}$. The enzyme digestion was stopped by cell culture medium with FBS, and the cells were washed down using pipettes. The suspensions were centrifuged and the supernatant medium with trypsinase was removed. Then cell pellets were resuspended and diluted in cell culture medium to a density of $10^{6}$ per $\mathrm{ml}$.

The sterilized aligned Si-NW arrays put in sterile Petri dishes were immerged into cell culture medium $(6 \mathrm{~mL} /$ dish $)$ in advance. Suspended cells were added into cell culture medium and shaken slightly to help dispersion, and then the cells were incubated at $37{ }^{\circ} \mathrm{C}$ in $5 \% \mathrm{CO}_{2}$. The aligned $\mathrm{Si}-$ NW arrays were taken out of cell culture medium at 2, 6, 12,24 , and $36 \mathrm{~h}$ and the cells attached on them were fixed. To maintain the morphology, the size, and the essential state of the cells for observation and measurement, the samples were fixed with $2.5 \%$ glutaraldehyde and $4 \%$ paraformaldehyde (in $1 \times$ PBS) for $2 \mathrm{~h}$ for fixation, ${ }^{14,19}$ and then further rinsed three times with PBS buffer. Dehydration and a critical point dry technique were carried out, as described in previous literatures, ${ }^{32,33}$ after immerging samples in $1 \%$ chlorauric acid 


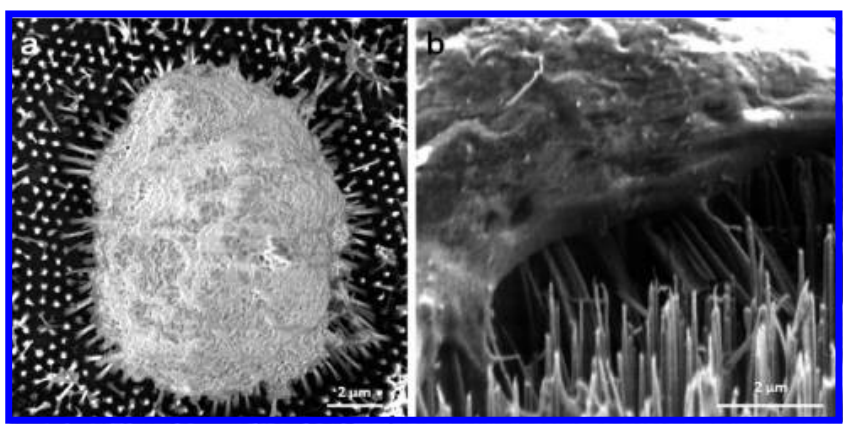

Figure 2. SEM images of mechanocyte bending aligned Si-NW arrays. (a) A top view image of a normal cell, a mechanocyte, layinig on uniform Si NW arrays. Si NWs at the edge are bent toward the center of the cell. The cell was cultured on the substrate for $24 \mathrm{~h}$. (b) A side view of the bent Si NWs under contraction of a mechanocyte cultured for $24 \mathrm{~h}$. The tips of the Si NWs have been adhered by the cell.

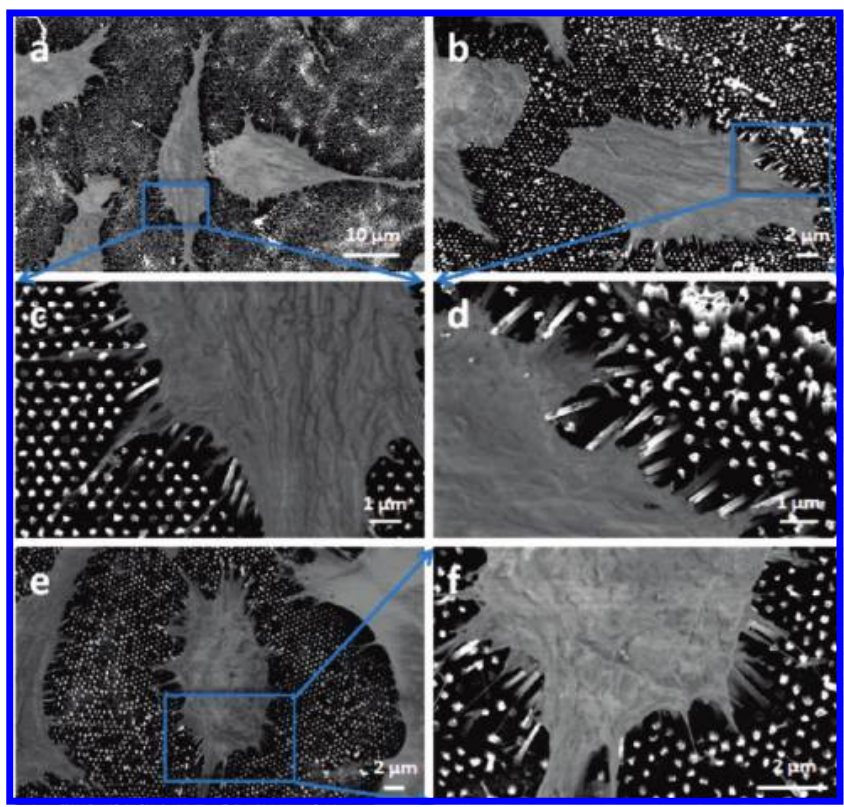

Figure 3. Measurement of the lateral deflections of Si-NWs as a result of the contraction force of a cancer cell. (a,c). L929 cell cultured on aligned Si-NWs for $24 \mathrm{~h}$. (c) An enlarged section of the image in panel a in which the lateral deflections $d$ were measured for different NWs. (b,d) L929 cell cultured on aligned Si-NWs for $36 \mathrm{~h}$. (e,f) SEM images of L929 cells cultured for $24 \mathrm{~h}$ on Si-NWs.

(in DI water) for $2 \mathrm{~h}$ for contrast enhancement. The entire procedures are operated strictly for protecting the samples to preserve their original morphology and state. All specimens were observed and recorded by SEM (Figures 2a,b and $3 a-f)$. After checking by SEM, Si-NWs with smaller diameters either in the group of $140 \pm 16.7 \mathrm{~nm}$ or in the group of $280 \pm 40.4 \mathrm{~nm}$ (aspect ratio is $21.7 \pm 2.6$ ) were used for the experiments, because they were more easily bent by the cells.

Measurement Method. Figure 2 shows SEM images of mechanocytes lying on top of aligned Si-NW arrays. The bending of the NWs at the edge of the cell can be clearly imaged (Figure 2a). The bending of the NWs underneath the cell can be seen at the side view of the cell (Figure $2 b$, Supporting Information, Figure S3). The typical bending angle of the NWs is no more than $30-40^{\circ}$, which is beyond the range of linear mechanics, but still in the elastic range of NWs, which can bear a bend over $90^{\circ}$ without any fracture or plastic deformation. In such a contact, the CTF acts right at the tip of the NW along the lateral direction parallel to the substrate. This is the geometry that produces the largest NW deflection and highest measurement sensitivity and accuracy. Figure 3 shows the observations of L929 cells cultured for 24 and $36 \mathrm{~h}$. The degree of bending of the NWs is apparent. It is known from the mechanical behavior of the NWs that the force required to bend a NW can be derived from the degree of its bending, provided the force-displacement relationship is quantitatively established.

Atomic force microscopy (AFM) was used to quantify the mechanical behavior of the NWs following a procedure previously described $^{34}$ (Supporting Information, Figure S2). By scanning the AFM tip across the tips of the NWs in contact mode, the elastic modulus of the NWs was derived by correlating the force applied parallel to the substrate (or nearly perpendicular to the NW) and the measured lateral bending distance parallel to the substrate. From the maximum bending distance and the corresponding bending force, the elastic modulus of the corresponding $\mathrm{Si} \mathrm{NW}$ is

$$
E=K L^{3} / 3 I
$$

provided the bending is in linear range, where $K=f / x$ is the spring constant of the Si NW, $f$ is the maximum bending force that is received from the lateral force image; $x$ is the corresponding bending distance that could be retrieved from the topography image; $L$ is the length of the NW; and $I$ is the momentum of inertia of the Si NW. If the NW has a uniform circular cross-section along its length, the momentum of inertia is

$$
I=\frac{\pi}{4} \cdot a^{4}
$$

where $a$ is also the radius of the NW. The advantage of this technique is that the elastic modulus of individual Si NWs can be measured systemically and simultaneously without destructing the sample.

The elastic modulus of the as-fabricated Si-NW arrays was first measured before cell culturing. The measurement was carried by AFM (MFP-3D from AsylumResearch Co. Ltd.). The cantilever was made of $\mathrm{Si}$ with a calibrated normal spring constant of $1.6 \mathrm{~N} / \mathrm{m}$ while the lateral spring constant was $249.1 \mathrm{~N} / \mathrm{m}$. The well vertically aligned Si NW arrays were scanned by AFM in contact mode with a normal force of $100 \mathrm{nN}$. Scanning speed was set at $30 \mu \mathrm{m} / \mathrm{s}$. Both the topography and lateral images were recorded. As presented in ref 34 , from the topography image one scanning line is picked up to calculate the bending distance while the corresponding lateral force curve gives the bending force caused by the bending of the NW. After 10 group measurements using the Si-NWs that have a uniform cylindrical geometry (Supporting Information, Figure S2), the average elastic modulus of Si NW was received to be $151 \pm 38.3$ GPa. 


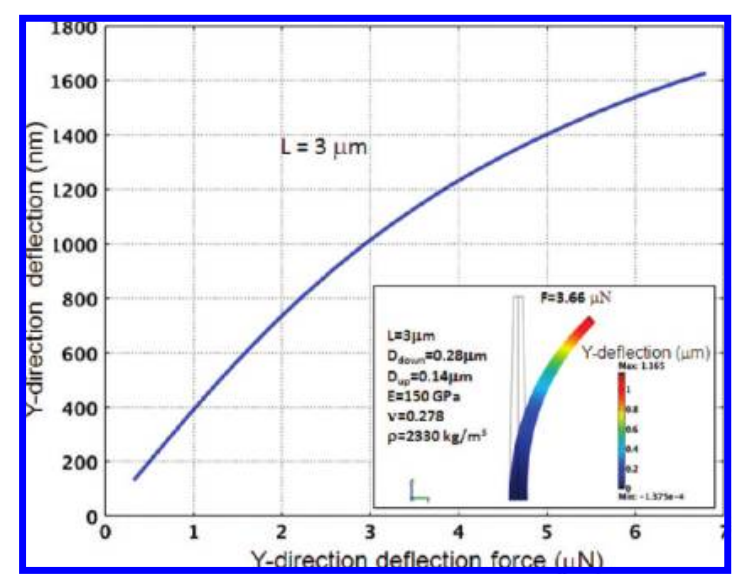

Figure 4. FEM calculated relationship between the applied force parallel to the substrate ( $y$-direction) and the tip deflection distance of a Si-NW. The schematic diagram in the right-bottom corner shows the deformation of Si-NW. The NW in the FEM is $3 \mu \mathrm{m}$ in length with a bottom diameter of $0.28 \mu \mathrm{m}$ and a top diameter of $0.14 \mu \mathrm{m}$. Young's modules $E=150 \mathrm{GPa}$ and Poisson's ratio $=$ 0.278 . The color code represents the lateral displacement.

The lateral displacement $d$ of a NW parallel to the substrate was measured directly from the top-view SEM image using software Image $\mathrm{J} 1.37 \mathrm{v},{ }^{35}$ and it will be used to derive the CTF parallel to the substrate. The cell spreading area on the substrate was also measured using this software.

To derive the CTF, we must consider the nonlinear mechanical effect of the NW because the degree of its bending is rather large. For this purpose, Finite Element (FEM) simulations were carried out in order to derive the relationship between the applied transverse force and the corresponding tip displacement for a Si-NW (Figure 4) using the experimentally measured dimensions and mechanical parameters. Our calculation was based on COMSOL package including nonlinear mechanical effects. Considering the realistic shape of the NW, its shape was modeled as a truncated cone with a bottom diameter of $0.28 \mu \mathrm{m}$ and a top diameter of $0.14 \mu \mathrm{m}$. Three different lengths have been taken into account, 2, 2.5, $3 \mu \mathrm{m}$, in accordance with SEM characterizations $(2.5 \mu \mathrm{m}$ in Supporting Information Figure S4). The mechanical properties for silicon were chosen as follows: Young's modulus $E=150 \mathrm{GPa}$ and Poisson's ratio $=0.278$. The base of the NW was fixed to a flat substrate and the force was applied in parallel to the substrate at the top surface of the NW. Figure 4 shows a calculated curve for a free-standing beam, in which the bending configuration of the beam is inset. This curve serves as the calibration for quantifying the transverse force from the measured lateral displacement of the NW tip. The results for different cells will be presented in Figures 5-7.

Result and Discussion. In this study, the Si-NWs are rigid and their Young's modules $(E=150 \mathrm{GPa})$ are the highest of all the materials used for investigating the CTF, which is close to the Young's modules of bulk silicon material. The maximal CTFs of three different cells are in the microNewton range. The average values of the CTF for the mechanocyte, HeLa cell, and L929 cell are $2.32 \pm 0.16,2.84 \pm 0.49$, and $3.48 \pm 0.46 \mu \mathrm{N}$, respectively, being cultured for $24 \mathrm{~h}$. Their maxima are up to $2.6,4.0 \mu \mathrm{N}$ and $4.76 \mu \mathrm{N}$, respectively

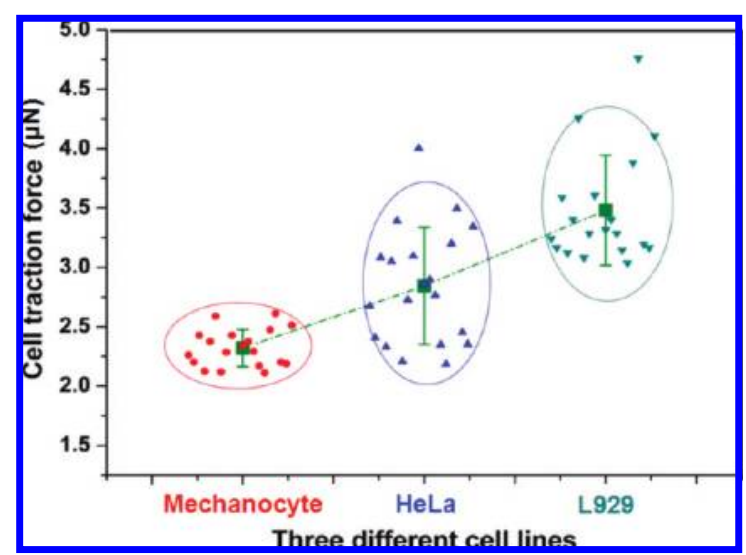

Figure 5. The measured maximum and average CTFs of mechanocyte, HeLa cell and L929 cell, each of which was cultured for $24 \mathrm{~h}$. The data points were plotted with a slight shift in the horizontal axis to show the number of data points. The green dots represent the averages of the corresponding groups. The vertical bar represents the standard deviation.

(Figure 5). These three cell lines were chosen for representing the following three distinct groups: normal mammalian cells, benign cells, and malignant cells. Since the mechanocyte and L929 cells were from the same histogenesis, the comparison between the two may give us a clue about their CTFs with the changing of gene expression. The average CTFs of the HeLa cell and the L929 cell are increased by 22 and 50\% more than that of the mechanocyte, respectively. Their corresponding maximal CTFs are increased by 53 and $83 \%$, respectively. From this result, we may conclude that the CTFs of the L929 cells and HeLa cells are significantly higher than that of the mechanocyte, and the variation trends of the CTFs (mean and maximum) from mechanocyte to HeLa cell and L929 cell are similar. Such results are received for the first time for normal cells, benign cells and malignant cells.

The relationships between CTFs and cell adhesion time have been observed for mechanocyte and L929 cells. From the specimen kept in cell culture medium for 2 to $36 \mathrm{~h}$, the CTFs have been measured (Figure 6). The CTF of the mechanocyte varies between 2.7 to $4.3 \mu \mathrm{N}$ when cultured from 2 to $36 \mathrm{~h}$. However, the CTF values of the L929 cell are between 1.77 to $6.2 \mu \mathrm{N}$. The CTF of the mechanocyte is relatively stable, while the CTF of the L929 is significantly larger. A larger CTF of the L929 cells may indicate their higher migration ability, consistent with the general observation about L929 cells.

A question that one may ask is if there is any connection between the cell spreading area and the CTF. Though there are some discussions about both, ${ }^{13,14}$ the relationship is still not clear. We measured the cell extending area and the corresponding CTF for L929 cells as a function of culture time (Figure 7). The cell's spreading area increases with cell growth time, although there is a small dip from 6 to $12 \mathrm{~h}$. The CTF curve first dropped and then increased. The trends of the two curves are analogous.

In comparison to the data reported in ref 14 using the microposts, the CTF measured by our technique is much higher. We believe that our data are more reasonable for the following reasons. First, our measurement used the 


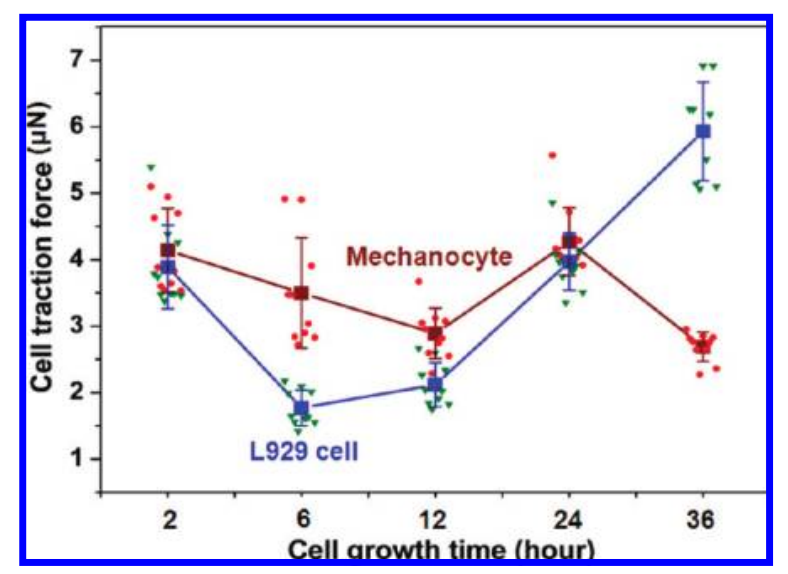

Figure 6. Relationships between the CTFs and cell adhesion time for the mechanocyte and L929 cells cultured from 2 to $36 \mathrm{~h}$. The data points were plotted with a slight shift in horizontal axis to show the number of data points. The lines represent the trends of the averages of the CTFs. The vertical bar represents the standard deviation.

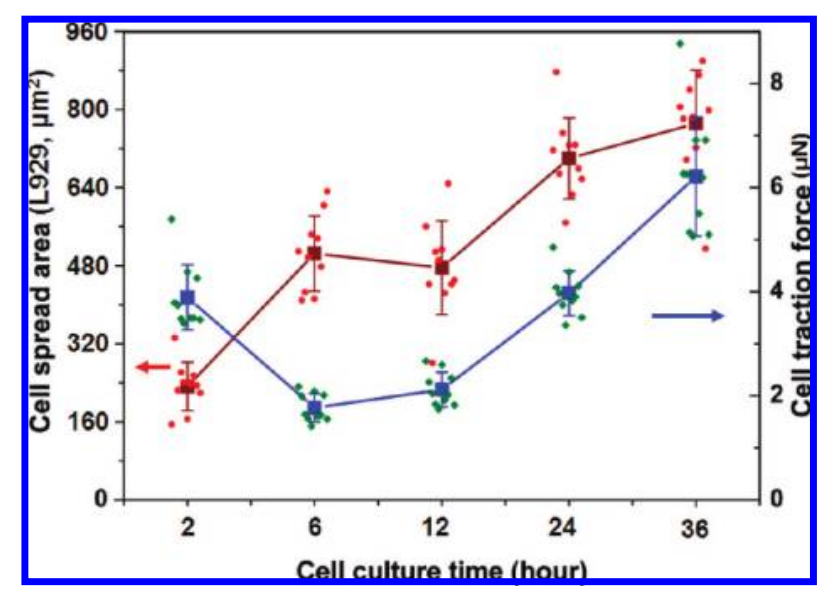

Figure 7. The normalized relationship of CTFs and cell spread area by L929 cells as a function of culturing time. The data points were plotted with a slight shift in horizontal axis to show the number of data points. The lines represent the trends of the averages of the cell spread areas. The vertical bar represents the standard deviation.

nanowires with a high Young's modulus and a high aspect ratio, which were grown on a solid and rigid substrate. Such nanowires have little self-elongation in comparison to the microposts made using polymer, significantly increasing the reliability of the measured data. In contrast, microposts made using polymer on a soft substrate may suffer from elongation and a vertical pulling effect once in interaction with cells. Saez et al. ${ }^{8,36}$ and Ghibaudo et al. ${ }^{21}$ reported that cell mechanics strongly depend on the rigidity of the substrate, and that the CTFs are proportional to the substrate rigidity provided that the deformation remains constant. Tan et al. ${ }^{14}$ and Nobes and Hall ${ }^{37}$ suggested the existence of two classes of force-adhesion size relationships. Second, the density of the Si-NWs $\left(\sim 4 \times 10^{8} \mathrm{NWs}\right.$ per $\left.\mathrm{cm}^{2}\right)$ is much higher than that of the microposts $\left(\sim 3 \times 10^{7}\right.$ microposts per $\left.\mathrm{cm}^{2}\right) .{ }^{20}$ As a result, the cell can sink to the bottom of the microposts onto the substrate due to the large intermicro-post distance, so that the force acting on the microposts is close to their roots. The largely decreased torque acting on the microposts resulted in small lateral deflection, thus, the force was probably underestimated and the experimental error could be large as well. In our measurement, we made sure that the density of the NWs was high enough so that the cells were at the top of the NWs, which was beneficial for data analysis and the high sensitivity of the force measurement. Furthermore, the large aspect ratio and small size of our NW largely increased the degree of bending and the accuracy of measurements.

In summary, utilizing the aligned $\mathrm{Si}-\mathrm{NW}$ arrays as nanoprobes, we present a method for quantifying the maximal cell traction forces (CTFs) of three different cell lines, normal mammalian cells, benign cells (L929), and malignant cells $(\mathrm{HeLa})$. By culturing the cells on a Si-NW array, the CTF of a cell results in an in-ward bending of NWs. By quantifying the force required to induce the bending of a NW based on the calibrated mechanical parameters provided by AFM and finite element calculation, the CTF of the cell acting on a NW is measured. We found that the cancer cells exhibit significantly larger CTFs in comparison to the normal cell. Discussions are presented regarding the reliability of the force data and the corresponding sensitivity. Our method and results are potentially useful for oncology, disease diagnosis, drug developing, and tissue engineering.

Acknowledgment. Research supported by DARPA (Army/ AMCOM/REDSTONE AR, W31P4Q-08-1-0009), BES DOE (DE-FG02-07ER46394), KAUST Global Research Partnership, Emory-Georgia Tech CCNE from NIH, and NSF (DMS 0706436, CMMI 0403671). Z.L. and H.F. are joint students between Georgia Tech and Peking University, and they thank the partial fellowship support by the China Scholarship Council (CSC) (No. 20073020) that supported them to carry out the research at Georgia Tech. M.-Y.L. was on a ROC National Science Council Fellowship for conducting research at Georgia Tech.

Supporting Information Available: Fabrication procedures of Si nanowire arrays and procedure for using Si-NW arrays to measure the CTFs of live cells in real time. This material is available free of charge via the Internet at http:// pubs.acs.org.

\section{References}

(1) Addae-Mensah, K. A.; WIKSWO, J. P. Exp. Biol. Med. 2008, 233, 7.

(2) Cross, S. E.; Jin, Y. S.; Rao, J. Y.; Gimzewski, J. K. Nat. Nanotechnol. 2007, $2,12$.

(3) Wang, J. H.-C.; Lin, J.-S. Biomech. Model Mechanobiol 2007, 6, 6.

(4) (a) Mussig, E.; Steinberg, T.; Schulz, S.; Spatz, J. P.; Ulmer, J.; Grabe, N.; Kohl, A.; Komposch, G.; Tomakidi, P. Adv. Funct. Mater. 2008, 18, 19. (b) Saez, A.; Ghibaudo, M.; Buguin, A.; Silberzan, P.; Ladoux, B. Proc. Natl. Acad. Sci. U.S.A. 2007, 104, 20. (c) Delanoe-Ayari, H.; Iwaya, S.; Maeda, Y. T.; Inose, J.; Riviere, C.; Sano, M.; Rieu, J. P. Cell Motil. Cvtoskeleton 2008, 65, 4. (d) Cai, Y. F.; Biais, N.; Giannone, G.; Tanase, M.; Jiang, G. Y.; Hofman, J. M.; Wiggins, C. H.; Silberzan, P.; Buguin, A.; Ladoux, B.; Sheetz, M. P. Biophvs. J. 2006, 91, 10 .

(5) Balaban, N. Q.; Schwarz, U. S.; Riveline, D.; Goichberg, P.; Tzur, G.; Sabanay, I.; Mahalu, D.; Safran, S.; Bershadsky, A.; Addadi, L.; Geiger, B. Nat. Cell Biol. 2001, 3, 5.

(6) Choquet, D.; Felsenfeld, D. P.; Sheetz, M. P. Cell 1997, 88, 1.

(7) Sniadecki, N. J.; Desai, R. A.; Ruiz, S. A.; CHEN, C. S. Ann. Biomed. Eng. 2006, 34, 1. 
(8) Zamir, E.; Katz, M.; Posen, Y.; Erez, N.; Yamada, K. M.; Katz, B.Z.; Lin, S.; Lin, D. C.; Bershadsky, A.; Kam, Z.; Geiger, B. Nat. Cell Biol. 2000, 2, 4.

(9) Wang, J. X.; Zohar, R.; McCulloch, C. A. Exp. Cell Res. 2006, 312 3.

(10) Miyamoto, S.; Teramoto, H.; Gutkind, J. S.; Yamada, K. M. J. Cell Biol. 1996, 135, 6 .

(11) Zamir, E.; Katz, B. Z.; Aota, S. I.; Yamada, K. M.; Geiger, B.; Kam, Z. J. Cell Sci. 1999, 112, 11.

(12) Yamada, K. M.; Geiger, B. Curr. Opin. Cell Biol. 1997, 9, 1.

(13) Tolic-Norrelykke, I. M.; Wang, N. J. Biomech. 2005, 38, 7.

(14) Tan, J. L.; Tien, J.; Pirone, D. M.; Gray, D. S.; Bhadriraju, K.; Chen, C. S. Proc. Natl. Acad. Sci. U.S.A. 2003, 100, 4.

(15) (a) Marshall, B. T.; Long, M.; Piper, J. W.; Yago, T.; McEver, R. P.; Zhu, C. Nature 2003, 423, 6936. (b) Merkel, R.; Nassoy, P.; Leung, A.; Ritchie, K.; Evans, E. Nature 1999, 397, 6714. (c) Benoit, M.; Gabriel, D.; Gerisch, G.; Gaub, H. E. Nat. Cell Biol. 2000, 2, 6.

(16) (a) Bell, E.; Ivarsson, B.; Merrill, C. Proc. Natl. Acad. Sci. U.S.A 1979, 76, 3. (b) Campbell, B. H.; Clark, W. W.; Wang, J. H. J. Biomech. 2003, 36, 1. (c) Ferrenq, I.; Tranqui, L.; Vailhe, B.; Gumery, P. Y.; Tracqui, P. Acta Biotheor. 1997, 45, 3-4.

(17) (a) Harris, A. K.; Stopak, D.; Wild, P. Nature 1981, 290, 5803. (b) Burton, K.; Taylor, D. L. Nature 1997, 385, 6615.

(18) Galbraith, C. G.; Sheetz, M. P. Proc. Natl. Acad. Sci. U.S.A. 1997, 94, 17.

(19) Roure, O. D.; Saez, A.; Buguin, A.; Austin, R. H.; Chavrier, P.; Silberzan, P.; Ladoux, B. Proc. Natl. Acad. Sci. U.S.A. 2005, 102, 7.

(20) (a) Yang, M. T.; Sniadecki, N. J.; Chen, C. S. Adv. Mater. 2007, 19, 20. (b) Xiang, Y.; LaVan, D. A. Appl. Phys. Lett. 2007, 90, 13. (c) Chandra, D.; Taylor, J. A.; Yang, S. Soft Matter 2008, 4, 5. (d) Milner, K. R.; Siedlecki, C. A. J. Biomed. Mater. Res., Part A 2007, 82A, 1. (e) Yang, Z. C.; Linb, J. S.; Chen, J. X.; Wang, J. H.-C. J. Theor. Biol. 2006, 242, 3.

(21) Ghibaudo, M.; Saez, A.; Trichet, L.; Xayaphoummine, A.; Browaeys, J.; Silberzan, P.; Buguinb, A.; Ladoux, B. Soft Matter 2008, 4, 9.

(22) Das, T.; Maitia, T. K.; Chakraborty, S. Lab Chip 2008, 8, 8.

(23) Li, B.; Xie, L.; Starr, Z. C.; Yang, Z. C.; Lin, J. S.; Wang, J. H.-C. Cell Motil. Cytoskeleton 2007, 64, 7.

(24) Schwarz, U. S.; Balaban, N. Q.; Riveline, D.; Bershadsky, A.; Geiger, B.; Safran, S. A. Biophys. J. 2002, 83, 3.

(25) Patolsky, F.; Timko, B. P.; Yu, G.; Fang, Y.; Greytak, A. B.; Zheng, G.; Lieber, C. M. Science 2006, 313, 5790.

(26) Cohen-Karni, T.; Timko, B. P.; Weiss, L. E.; Lieber, C. M. Proc. Natl. Acad. Sci. U.S.A. 2009, 106, 18.

(27) Timko, B. P.; Cohen-Karni, T.; Yu, G.; Qing, Q.; Tian, B.; Lieber, C. M. Nano Lett. 2009, 9, 2.

(28) Patolsky, F.; Zheng, G.; Lieber, C. M. Anal. Chem. 2006, 78, 13.

(29) Lieber, C. M.; Wang, Z. L. MRS Bull. 2007, 32, 99-104.

(30) Patolsky, F.; Zheng, G.; Lieber, C. M. Nanomedicine 2006, 1, 1.

(31) Huang, Z.; Fang, H.; Zhu, J. Adv. Mater. 2007, 19, 744.

(32) Kim, W.; Ng, J. K.; Kunitake, M. E.; Conklin, B. R.; Yang, P. D. J. Am. Chem. Soc. 2007, 129, 23.

(33) Li, Z.; Yang, R. S.; Yu, M.; Bai, F.; Li, C.; Wang, Z. L. J. Phys. Chem. C 2009, 112, 51.

(34) Song, J. H.; Wang, X. D.; Riedo, E.; Wang, Z. L. Nano Lett. 2005, 5, 10.

(35) NIH, U.S.A, http://rsb.info.nih.gov/ij/ Java 1.5.0_09.

(36) Saez, A.; Buguin, A.; Silberzan, P.; Ladoux, B. Biophys. J. 2005, 89,6 .

(37) Nobes, C. D.; Hall, A. Cell 1995, 81, 1.

\section{NL901774M}

